\title{
ON VISCOUS AND RESISTIVE DISSIPATION OF ALFVÉN WAVES IN AN ISOTHERMAL ATMOSPHERE
}

\author{
HADI YAHYA ALKAHBY \\ Department of Mathematics and Computer Science \\ Dillard University \\ New Orleans, LA 70122, USA
}

(Received June 30, 1995 and in revised form November 10, 1995)

\begin{abstract}
In this articie we will investigate the refiection and dissipation of Alfvern waves, resulting from a uniform vertical magnetic field, in a viscous, resistive and isothermal atmosphere. It is shown that the atmosphere may be divided into two distinct regions connected by an absorbing and reflecting transition layer. In the transition layer the reflection, dissipation and absorption of the magnetic energy of the waves take place and in it the kinematic viscosity changes from small to large values. In the lower region the effect of the resistive diffusivity and kinematic viscosity changes from small to large values. In the lower region the effect of the resistive diffusivity and kinematic viscosity is negligible and in it the solution can be represented as a linear combination of two, incident and reflected, propagating waves with different wavelengths and different dissipative factors. In the upper region the effect of the resistive diffusivity and kinematic viscosity is large and the solution, which satisfies the prescribed boundary conditions, will behave as a constant. The reflection coefficient, the dissipative factors are determined and the conclusions are discussed in connection with solar heating
\end{abstract}

KEY WORDS AND PHRASES: Alfvén Waves, Atmospheric Waves, Wave Propagation. 1991 AMS SUBJECT CLASSIFICATION CODES: 76N, 76Q.

\section{INTRODUCTION}

It is well known that the solar corona is extremely hot, the typical temperature is $2 \times 10^{6} \mathrm{~K}$, at heights in excess of $2000 \mathrm{Km}$, compared with $5 \times 10^{3} \mathrm{~K}$, temperature minimum, in the atmosphere (see Priest [3] for references). As a result, thermal energy must be continually supplied to maintain this temperature against radiative cooling. The old ideas for coronal heating were that of sound waves being generated in the convection zone that could propagate through the solar chromosphere, steeping into shocks and to give global heating. Sound waves have been ruled out in connection with coronal heating, however, because their low group velocity means that they cannot supply the necessary energy However, the remnants of this idea remain. Recent theories of the solar heating invoke, strongly, the magnetic energy dissipation as a source of thermal energy. In particular, recent investigations are focusing on the dissipation of magnetic energy, resulting from a vertical magnetic field, and its role in the heating process of the solar atmosphere.

The aim of this paper is to investigate the combined effect of the electrical conductivity and the viscosity on the reflection and dissipation of Alfven waves, resulting from a uniform vertical magnetic field, in an isothermal atmosphere. It is shown that if the effect of the viscosity is small compared to that of the resistive diffusivity the atmosphere can be divided into two different regions. In the lower region the influence of the resistive diffusivity and kinematic viscosity is negligible and the solution can be written as a linear combination of incident and reflected waves with different wavelengths and different dissipating factors. In fact the wavelength factor of the reflected wave is smaller than that of the incident 
wave. This indicates that the magnetic energy which dissipates from the incident wave is larger than that of the reflected wave and the difference in the magnetic energy of the reflected and the incident waves represents the dissipated energy in the transition region. Moreover, the dissipative factors are a function of the electrical resistivity and the dynamic viscosity. In the upper region the effect of the resistive diffusivity and the kinematic viscosity is large. As a result, the solution, which satisfies the dissipation condition, behaves as a constant. The lower and the upper regions are connected by a transition layer in which the kinematic viscosity changes from small to large values because of the exponential decrease of the density with height. Moreover, in the transition region the reflection and dissipation of Alfven waves take place. The differences in both, wavelengths and dissipating factors, result from the absorption and dissipation process of the magnetic energy in the transition layer. the reflection coefficient and the dissipative factors are determined. The conclusions are discussed in connection with the process of the heating of the solar atmosphere.

\section{MATHEMATICAL FORMULATION OF THE PROBLEM}

We will consider an isothermal atmosphere, which is viscous and resistive, and occupies the upper half-space $z>0$. It will be assumed that the gas is thermally non-conducting and under the influence of a uniform vertical magnetic field. We will investigate the problem of small oscillations about equilibrium, i.e. oscillations which depend only on time $t$, on the vertical coordinate $z$ and on the horizontal coordinate $x$.

Let the equilibrium pressure, density, temperature and magnetic field strength be denoted by $P_{0}(x)$, $\rho_{0}(x), T_{0}$, and $\mathbf{B}_{0}(\mathbf{x})$, where $P_{0}(x), \rho_{0}(x)$ and $T_{0}$ satisfy the gas law $P_{0}(x)=R T_{0} \rho_{0}(x)$ and the hydrostatic equation $P_{0}^{\prime}(x)+g \rho_{0}(x)=0$. Here $R$ is the gas constant, $g$ is the gravitational acceleration and the prime denotes differentiation of the pressure with respect to $x$. The equilibrium pressure and density,

$$
P_{0}(x)=P_{0}(0) \exp (-x / K), \quad \rho_{0}(x)=\rho_{0}(0) \exp (-x / K),
$$

where $K=R T_{0} / g$ is the density scale height.

Let $p(x, z, t), \rho(x, z, t), V(x, z, t)$, and $\mathbf{h}(\mathbf{x}, \mathbf{z}, \mathbf{t})$ be the perturbations quantities in the pressure, density, velocity, and the magnetic field strength.

Alfvén waves are incompressible because they have motions transverse to the magnetic field, i.e. they do not couple to slow or fast magnetohydrodynamics waves in a homogeneous medium, Priest [3]. As a result, they can be described only by the induction and momentum equations and dissipation of linear waves is not affected by thermal conduction or radiation. Thus, the equations of motion are

$$
\begin{gathered}
\frac{\partial \mathbf{H}}{\partial \mathbf{t}}+\nabla \mathbf{x}(\mathbf{H x \mathbf { V }})=-\nabla \mathbf{x}\left[\left(\frac{c}{4 \pi \mu \sigma}\right) \nabla \mathbf{x H}\right], \\
\begin{aligned}
\rho_{0}\left[\frac{\partial \mathbf{V}}{\partial \mathbf{t}}\right. & +(\mathbf{V} \cdot \nabla) \mathbf{V}]+\nabla \mathbf{p}-\mathbf{g} \rho+\frac{\mu}{4 \pi}[\mathbf{H x}(\nabla \mathbf{x H})] \\
& =\nu \nabla^{2} \mathbf{V}+(\nabla \nu \cdot \nabla) \mathbf{V}+\nabla\left[\nu^{-}(\nabla \cdot \mathbf{V})\right],
\end{aligned}
\end{gathered}
$$

where $\mathbf{H}(\mathbf{x}, \mathbf{z}, \mathbf{t})=\mathbf{B}(\mathbf{x}) \mathbf{e}_{\mathbf{z}}+\mathbf{h}(\mathbf{x}, \mathbf{z}, \mathbf{t}) \mathbf{e}_{\mathbf{y}}, \quad \nabla=\mathbf{e}_{\mathbf{x}} \frac{\partial}{\partial x}+\mathbf{e}_{\mathbf{y}} \frac{\partial}{\partial y}+\mathbf{e}_{\mathbf{z}} \frac{\partial}{\partial z}, \mathbf{V}(\mathbf{x})=v(x, z, t) \mathbf{e}_{\mathbf{y}}$ and $\mu$ is the permeability of the magnetic field, Alkahby [3,8]. Here $\nu$ and $\nu^{-}$are, respectively, the incompressible and compressible viscosities. Moreover, $c$ denotes the speed of light in vacuum and $\sigma$ is the Ohmic electrical conductivity. The induction equation (2.2) balances magnetic field oscillation, velocity transport along the magnetic field lines and compressibility against resistive dissipation by $\mathrm{Ohm}$ effect, the Hall effect being omitted. The momentum equation (2.3) balances the inertia force and pressure gradient against weight, magnetic and viscous forces. 
In this article we will consider the case of a uniform vertical magnetic field $\mathbf{B}_{0}(\mathbf{x})=\mathbf{B}_{0}$, as well as $\eta=\frac{c^{2}}{4 \pi \mu \sigma}$ and $\nu$, i.e. they are constants. It follows from equation $(2.1)$ that the kinematic viscosity and Alfvén speed can be written in the following form

$$
\begin{gathered}
\nu(x)=\left(\nu / \rho_{0}(0)\right) e^{x / K} \\
c_{A}(x)=c_{A}(0) e^{x / 2 K}
\end{gathered}
$$

where $c_{A}=\sqrt{\mu / 4 \pi \rho_{0}(0)} B_{0}$. Moreover, the linear forms of equations (2.2) and (2.3) are:

$$
\begin{gathered}
D_{t} h(x, z, t)-\eta\left[D_{z z} h(x, z, t)+D_{x x} h(x, z, t)\right]=B_{0} D_{z} v(x, z, t), \\
D_{t} v(x, z, t)-\nu(x)\left[D_{z z} v(x, z, t)+D_{x x} v(x, z, t)\right]=\left(c_{A}^{2} / B_{0}\right) D_{z} h(x, z, t) .
\end{gathered}
$$

In addition, the velocity $v(x, z, t)$ can be eliminated to obtain an equation for $h(x, z, t$,$) only This can$ be accomplished by differentiating equations (2.6) and (2.7) with respect to $t$ and using equation (2.7). The resulting differential equation is

$$
D_{t t} h(x, z, t)-c_{A}^{2} e^{x / K} D_{z z} h(x, z, t)=\left(\eta+\nu e^{x / K}\right) D_{t}\left[D_{x x} h(x, z, t)+D_{z z} h(x, z, t)\right] .
$$

BOUNDARY CONDITIONS. To complete the problem formulation certain boundary conditions must be imposed to ensure a unique solution. Since the gas is viscous and resistive the dissipation condition will be necessary and sufficient, as an upper boundary condition, to ensure a unique solution. the dissipation condition requires the finiteness of the rate of the energy dissipation in an infinite column of fluid of a unit cross-section. This implies,

$$
\int_{0}^{\infty}|R(x, k, t)|^{2} d x<\infty
$$

where $R(x, k, t)$ denotes the magnetic field spectrum of a wave with frequency $\omega$, wavelength $k$ and at the position $x$ (see equation 3.5). This boundary condition will not be applicable if $\nu=\sigma=0$, but it will be applicable if $\nu \neq 0$ or $\sigma \neq 0$. Moreover, a boundary condition is also required at $x=0$, and we shall set

$$
h(0, k, t)=1,
$$

by suitably normalizing $h(x, k, t)$. It will be seen that these boundary conditions will ensure a unique solution to within a multiplicative constant.

\section{SOLUTION OF THE PROBLEM}

In this section we will investigate the behavior of the solution of the differential equation (2.8) and we shall determine a series solution and obtain the asymptotic behavior of the solution. We can use Fourier representation in $z$ and $t$ for the magnetic field perturbation $h(x, z, t)$ because the properties of the atmosphere depend on $x$ only. In other words the magnetic field $h(x, z, t)$ is given by the following Fourier representation

$$
h(x, z, t)=\int_{-\infty}^{\infty} d \omega \int_{-\infty}^{\infty} R(x, k, t) \exp [i(k z-\omega t)] d k,
$$

where $R(x, k, t)$ denotes the magnetic field perturbation spectrum for a wave of frequency $\omega$, transverse wavenumber $k$ at a position $x$. Moreover, we will introduce the following dimensionless parameters,

$$
x^{\prime}=x / K, \quad \beta=k K, \quad \alpha_{1}=\omega K / c_{A}(0), \quad \alpha_{2}=\nu \omega / c_{A}^{2}(0), \quad \alpha_{3}=\eta \alpha_{2} / \nu, \quad \alpha=\alpha_{2} / \alpha_{3} .
$$

where the prime on the dimensionless variable $x$ is omitted for simplicity. After the substitution of equation (3.1) and the dimensionless parameters, defined in equation (3 2), into equation (2.8), we have the following differential equation 


$$
\left[1+e^{-x} / \alpha\right] D^{2} R(x, k, \omega)-\left[\beta^{2}\left(1-i \alpha_{2}\right)+\left(\beta_{2} / \alpha-i \alpha_{1} / \alpha_{2}\right) e^{-x}\right] R(x, k, \omega)=0,
$$

where $d=d / d x$. Since the solution will depend on $\rho / \alpha$, it will be convenient to introduce a new dimensionless variable $\chi$ defined by

$$
\chi=-e^{-x} / \alpha
$$

then the operator $D^{2}$ goes over into

$$
D^{2}=\chi^{2} d^{2} / d \chi^{2}+\chi d / d \chi
$$

For fixed value $\alpha>0$ the point $\chi=0$ corresponds to $x=\infty$, the point $\chi_{0}=-1 / \alpha$ to $x=0$ and the segment connecting these points in the complex $\chi$-plane to $x>0$. Moreover let

$$
R(\chi, k, \omega)=\chi^{r} \Phi(\chi)
$$

then differential equation (3.3) can be written in the following form where the prime denotes the derivative of $\Phi$ with respect to $\chi$,

$$
\chi(1-\chi) \Phi^{\prime \prime}(\chi)+[1+2 r-(1+2 r) \chi] \Phi^{\prime}-i\left(\alpha_{1}^{2} / \alpha_{3}-\beta^{2} \alpha_{2}\right) \Phi(x)=0,
$$

provided that the parameter $r$ satisfies the following relation

$$
r=\beta \sqrt{1-i \alpha_{2}}
$$

It is clear that he differential equation (3.3) is a special case of the hypergeometric equation

$$
\left[\chi(1-\chi) D^{2}+(c-(1+a+b) \chi) D-a b\right] \Phi(\chi)=0,
$$

with

$$
c=1-2 r, \quad a+b=2 r, \quad a b=i\left(\alpha_{1}^{2} / \alpha_{3}-\beta_{2} \alpha_{2}\right) .
$$

Moreover, equation (3.6) has three regular singular points, $\chi=0, \chi=1$, and $\chi=\infty$. The intermediate regular singular point $\chi=1$ corresponds to the reflecting layer. Solving for the dimensionless parameters $a$ and $b$ we have

$$
\begin{aligned}
& a=\beta\left[\sqrt{1-i \alpha_{2}}+\sqrt{1-i \alpha_{1}^{2} / \beta_{2} \alpha 3}\right], \\
& b=\beta\left[\sqrt{1-i \alpha_{2}}-\sqrt{1-i \alpha_{1}^{2} / \beta^{2} \alpha_{3}}\right] .
\end{aligned}
$$

For $|\chi|<1$, the hypergeometric equation (3.6) has two linearly independent solutions of the following form:

$$
\begin{gathered}
\Phi_{1}(\chi)=F(a, b, 2, \chi), \\
\Phi_{2}(\chi)=\chi^{-2 r} F(a-c+1, b-c+1,2-c, \chi),
\end{gathered}
$$

where

$$
F(a, b, 2, \chi)=\sum_{n=0}^{\infty} \frac{(a)_{n}\left(b_{n}\right.}{(c)_{n}} \cdot \frac{\chi^{n}}{n !}=\frac{\Gamma(c)}{\Gamma(a) \Gamma(b)} \sum_{n=0}^{\infty} \frac{\Gamma(a+n) \Gamma(b+n)}{\Gamma(c+n)} \cdot \frac{\chi^{n}}{n !} .
$$

For $|\chi|>1$ and $|\arg (-\chi)|<\pi$, the solution of equation (3.6) can be written in the following form:

$$
\begin{aligned}
& \Phi_{a}(\chi)=(-\chi)^{-a} F\left(a, 1-c+a, 1-b+a, \chi^{-1}\right), \\
& \Phi_{b}(\chi)=(-\chi)^{-b} F\left(b, 1-c+b, 1-a+b, \chi^{-1}\right) .
\end{aligned}
$$


The second solution $\Phi_{2}(\chi)$ will be eliminated by the boundary condition (2.9) because it increases to infinity as $x \rightarrow \infty$. As a result, the solution of the differential equation (3.6), which satisfies the dissipation condition, is a multiple of $\Phi_{1}(\chi)$, i.e.

$$
\Phi(\chi)=C \Phi_{1}(\chi)=C F(a, b, c \chi),
$$

where $C$ is a constant which can be determined from the boundary condition (2.10). For $|\chi|>1$, $|\arg (-\chi)|<\pi$, the analytic continuation of the solution of the differential equation (3.6) can be written like:

$$
\begin{aligned}
\phi(\chi)= & C\left[\frac{\Gamma(c) \Gamma(b-a)}{\Gamma(b) \Gamma(c-a)}(-\chi)^{-a} F\left(a, 1-c+a, 1-b+a, \chi^{-1}\right)\right. \\
& \left.+\frac{\Gamma(c) \Gamma(a-b)}{\Gamma(a) \Gamma(c-b)}(-\chi)^{-b} F\left(b, 1-c+b, 1-a+b, \chi^{-1}\right)\right]
\end{aligned}
$$

For $|\chi|>1$ and $|\arg (-\chi)|<\pi$ the asymptotic behavior of the solution, defined in equation (3.8), as $\alpha \rightarrow 0$ can be obtained by retaining the most significant terms in equation (3.18), the resulting equation is

$$
\Phi(\chi)=C\left[\frac{\Gamma(c) \Gamma(b-a)}{\Gamma(b) \Gamma(c-a)}(-\chi)^{-a}+\frac{\Gamma(c) \Gamma(a-b)}{\Gamma(a) \Gamma(c-b)}(-\chi)^{-b}\right]
$$

\section{MAGNITUDE OF THE REFLECTION COEFFICIENT AND CONCLUSIONS}

In this section we will investigate the behavior of the solution of the differential equation (3.6) defined by the hypergeometric function (3.17) and its asymptotic expansion defined by equation (3.19). In particular, we will determine the reflection coefficient and the dissipative factors To do this we have to make use of the dimensionless parameters $a$ and $b$. It is easy to see that

$$
\begin{aligned}
& a=\beta\left[\sqrt{1-i \alpha_{2}}+\sqrt{1-i \alpha_{1}^{2} / \beta^{2} \alpha_{3}}\right]=d_{1}-i d_{a}, \\
& b=\beta\left[\sqrt{1-i \alpha_{2}}-\sqrt{1-i \alpha_{1}^{2} / \beta^{2} \alpha_{3}}\right]=-d_{2}+i d_{b} .
\end{aligned}
$$

It is clear that $d_{1}>d_{2}$ and $d_{a}>d_{b}$. Reintroducing the original variable $x$ via (3.4), the equation (3.19) can be written in the following form

$$
\Phi(x) \sim C\left[\frac{\Gamma(c) \Gamma(a-b) \exp \left(-d_{2}+i d_{b}\right) \log \alpha}{\Gamma(a) \Gamma(c-b)}\right]\left[\exp \left[\left(-d_{2}+i d_{b}\right) x\right]+R \exp \left[\left(d_{1}-i d_{a}\right) x\right]\right],
$$

where $R$ denotes the reflection coefficient obtained from the ratio of the amplitudes of the reflected and incident waves and defined by

$$
R=\frac{\Gamma(a) \Gamma(c-b) \Gamma(b-a)}{\Gamma(b) \Gamma(c-a) \Gamma(a-b)} \exp \left(-2 \beta\left(\sqrt{1-i \alpha_{1}^{2} / \beta^{2} \alpha_{3}}\right) \log \alpha\right)
$$

The constant $C$ can be determined from the boundary condition (2.10). As a result,

$$
c=\frac{\Gamma(a) \Gamma(c-b) \exp \left(d_{2}-i d_{b}\right) \log \alpha}{\Gamma(c) \Gamma(a-b)[1+R]},
$$

and equation (4.3) can be written in the following form

$$
\Phi(x) \sim\left[\frac{1}{1+R}\right]\left[\exp \left[\left(-d_{2}+i d_{b}\right) x\right]+R \exp \left[\left(d_{1}-i d_{a}\right) x\right]\right] .
$$

As a consequence of the above results and discussion we have the following conclusions. 
[A] Equation (4.3) represents the behavior of the solution of the differential equation (3.6), which satisfies the prescribed boundary conditions, in the lower region. It indicates that the solution can be written as a linear combination of an incident and a reflected wave. In the upper region the solution, which satisfies the dissipation condition, should behave like a constant. These two regions are connected by a transition region.

[B] In the transition region the reflection, dissipation and modification of the waves takes place. Moreover, the kinematic viscosity changes from small to large values because of the exponential decrease of the density with $x$.

[C] The incident wave decays exponentially like $\exp \left(-d_{2} x\right)$, while the reflected wave decays like $\exp \left(-d_{1} x\right)$. Since $d_{1}>d_{2}$, the dissipative factor of the incident wave is larger than that of the reflected wave. On the contrary, the wavelength of the reflected wave $\lambda_{\tau}=2 \pi / d_{a}$ is smaller than that of the incident wave $\lambda_{\imath}=2 \pi / d_{b}$, because $d_{a}>d_{b}$. This indicates that the larger part of the dissipated magnetic energy comes from the incident wave.

[D] The dissipative factors are functions of the dynamic viscosity and electrical conductivity and they behave like exponentially decaying waves. This indicates that the magnetic energy of the wave dissipates as the wave propagates, upward and downward, but still most of the dissipation of the magnetic energy takes place in the transition region.

[E] The reflected wave, from the transition region, will be reflected upward at the boundary of $x=z=0$. Reflection and dissipation of the waves will continue, in the lower region, until the energy of the waves dissipates completely.

ACKNOWLEDGMENT. I would like to express my thanks to Professor Michael Yanowitch for his support and invaluable criticism during the preparation of this work.

\section{REFERENCES}

[1] LEROY, B., Propagation of waves in an atmosphere in the presence of a magnetic field (Part II: The reflection of the Alfvén waves), Astron. and Astrophys. 81 (1980), 136-146.

[2] ROBERTS, B.H., Waves in the solar atmosphere, Geophys. Astrophys. Fluid Dynam. 62 (1991), 83-100

[3] PRIEST, E.R., Solar Magnetohydrodynamics, D. Reidl Pub. Co., 1984

[4] ALKAHBY, H.Y., Acoustic-gravity waves in a viscous and thermally conducting isothermal atmosphere (Part I: For small Prandtl number), Internat. J. Math. and Math. Scl. 18, 2 (1995), 371-382.

[5] ALKAHBY, H.Y. and YANOWITCH, M., Reflection of vertically propagating waves in a thermally conducting isothermal atmosphere with a horizontal magnetic field, Geophys. Astrophys. Fluid Dynam. 56 (1991), 227-235.

[6] ALKAHBY, H.Y., On the coronal heating mechanism by the resonant absorption of Alfven waves, Internat. J. Math. and Math. Sci. 16, 4 (1993), 811-816.

[7] ALKAHBY, H.Y. and HUSSAIN, T., On the heating of the solar corona by the resonant absorption of Alfvén waves, Appl. Math. Lett. 6, 6 (1993), 59-63.

[8] ALKAHBY, H.Y., Reflection and dissipation of hydromagnetic waves in a viscous and thermally conducting isothermal atmosphere, Geophys. Astrophys. Fluid Dynam. 72 (1993), 197-207 


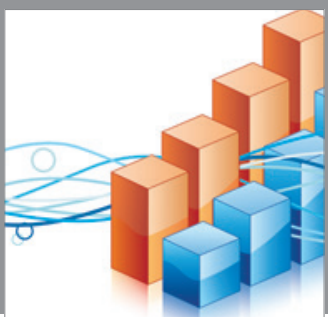

Advances in

Operations Research

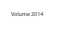

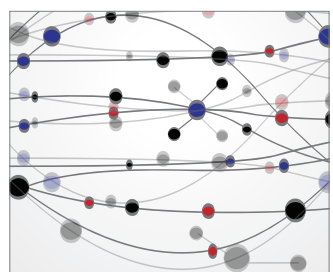

\section{The Scientific} World Journal
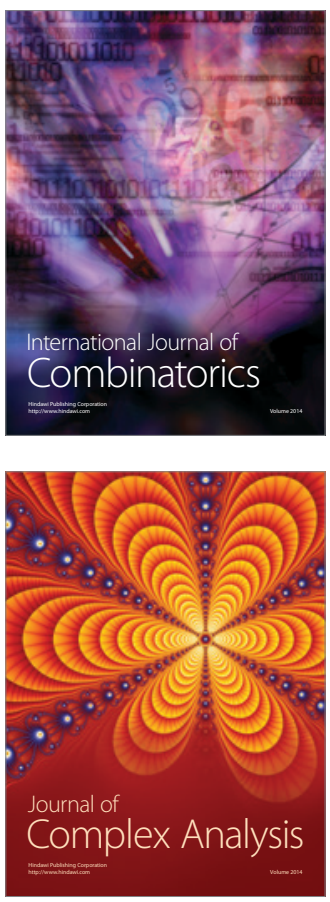

International Journal of

Mathematics and

Mathematical

Sciences
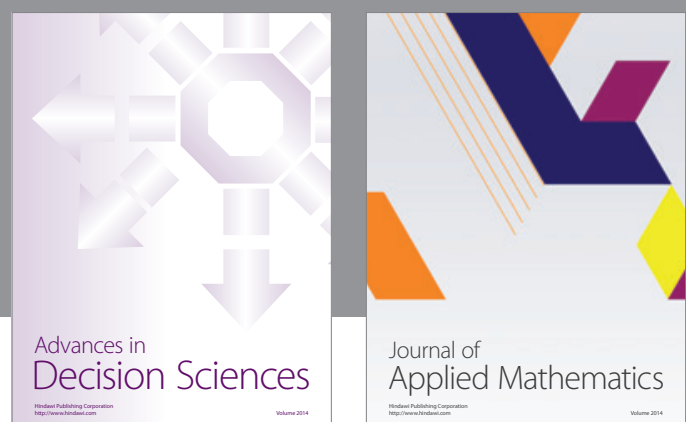

Journal of

Applied Mathematics
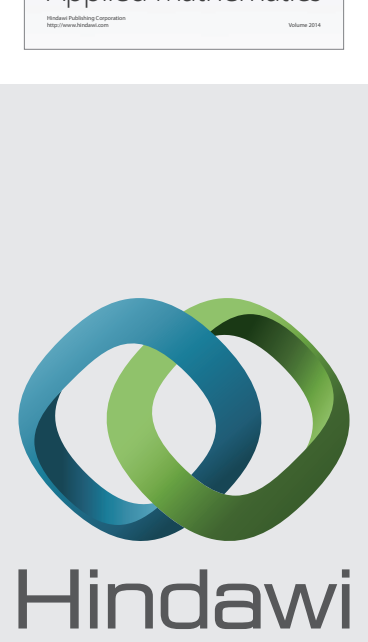

Submit your manuscripts at http://www.hindawi.com
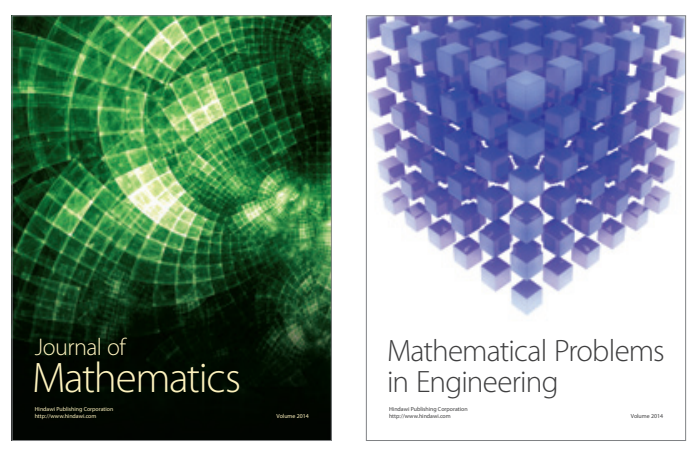

Mathematical Problems in Engineering
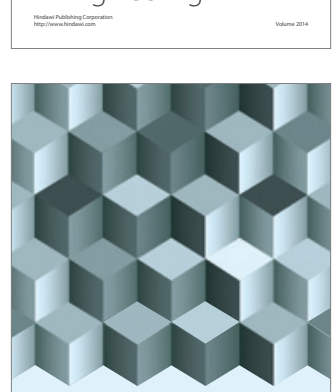

Journal of

Function Spaces
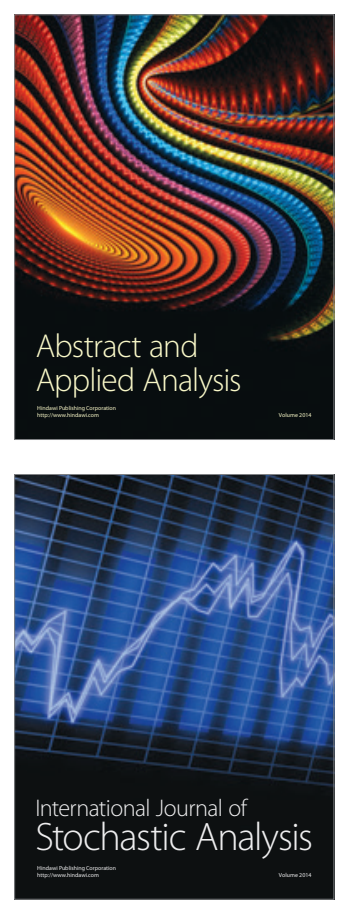

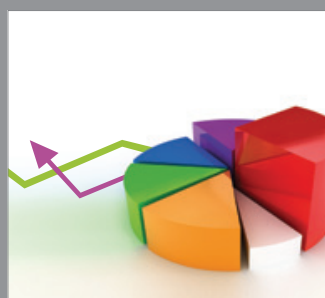

ournal of

Probability and Statistics

Promensencen
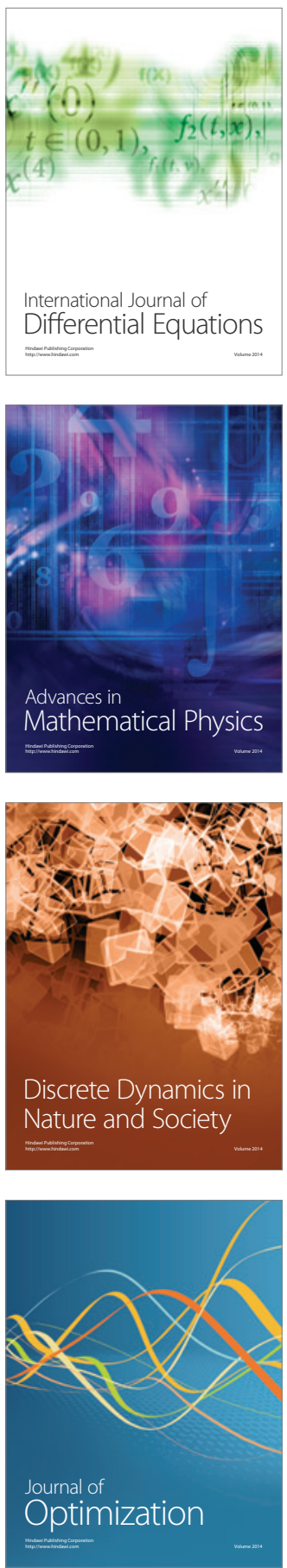